\title{
CONSTRUÇÃO DE MINI FORNO PARA FUNCIONALIZAÇÃo TÉRMICA DE FASES ESTACIONÁRIAS MONOLÍTICAS
}

\author{
Sandro Pereira Ribeiro ${ }^{a}$, José Paulo Rodrigues Furtado de Mendonça ${ }^{\mathrm{b}}$ e Marcone Augusto Leal de Oliveira ${ }^{\mathrm{a}, *, \text { (D) }}$ \\ aDepartamento de Química, Instituto de Ciências Exatas, Universidade Federal de Juiz de Fora, 36036-330 Juiz de Fora - MG, Brasil \\ bDepartamento de Física, Instituto de Ciências Exatas, Universidade Federal de Juiz de Fora, 36036-330 Juiz de Fora - MG, Brasil
}

Recebido em 11/09/2019; aceito em 06/11/2019; publicado na web em 05/02/2020

\begin{abstract}
CONSTRUCTION OF MINI OVEN FOR THERMAL FUNCTIONALIZATION OF MONOLITHIC STATIONARY PHASES. The present work describes a mini oven built to be applied in the preparation of monolithic stationary phase through the thermal polymerization approach. The developed device was successfully tested for functionalization of monolithic stationary phase in the internal surface of the silica capillary column fused with gold nanoparticles.
\end{abstract}

Keywords: stationary phase; monolithic columns preparation; thermopolymerization; labmade mini oven.

\section{INTRODUÇÃO}

O avanço de pesquisas na ciência de separação vem alcançando resultados cada vez mais expressivos, eficazes, eficientes e confiáveis, tanto para a identificação quanto para a quantificação de espécies químicas de interesse. As técnicas cromatográficas ou de eletromigração despontam como interlocutoras e mediadoras no processo de otimização e desenvolvimento dos métodos analíticos responsáveis pelas separações. Dentro desse contexto, o preparo de fases estacionárias (FE) para uso em técnicas de separação como cromatografia liquida de alta eficiência (CLAE), cromatografia gasosa (CG) e a eletrocromatografia capilar (ECC) continua sendo tema de relevante interesse. ${ }^{1,2}$

Dentre as técnicas elencadas, destaca-se a ECC, a qual é definida como técnica de separação que equaciona positivamente as características relevantes da CLAE, como o uso da FE, com a eletrosmose, fenômeno inerente a parede interna de tubos capilares de sílica fundida, comumente empregados em eletroforese capilar (EC) ${ }^{3}$ Os grupos silanóis presentes na parede interna da coluna capilar, a formação da dupla camada elétrica, o potencial zeta, o plano de cisalhamento, o $\mathrm{pH}$, a forca iônica e a viscosidade da solução em combinação com a aplicação de diferença de potencial (ddp) nas extremidades opostas do tubo capilar resultam em um fluxo induzido da fase móvel (FM), denominado de fluxo eletro-osmótico (FEO). ${ }^{4} \mathrm{O}$ FEO tem capacidade de impulsionar as bandas dos analitos, sob velocidade constante, através da estrutura porosa da FE, a qual se encontra devidamente ancorada em um pequeno intervalo da parede interna do capilar, e pode promover a separação dos constituintes a partir de interações especificas com os sítios ativos. A ECC é operacional em equipamento convencional de EC e apresenta vantagem interessante do ponto de vista da química verde, pois mitiga a necessidade do uso de solventes orgânicos nas análises. As aplicações envolvendo a ECC apresentam dependência direta com o tipo de FE e as funcionalizações usadas, o que amplia o preâmbulo possível de aplicações na área de separações, podendo englobar compostos ionizáveis, polares, apolares, isômeros de posição, de cadeia, geométricos e ópticos. Do ponto de vista assertivo e ilustrativo, é interessante destacar alguns trabalhos da literatura envolvendo o uso da ECC em análise de aminoácidos e peptídeos, ${ }^{5}$ proteínas, ${ }^{6}$ antraquinonas, ${ }^{7}$ hidrocarbonetos policíclicos aromáticos ${ }^{8}$ e racematos. ${ }^{9}$

*e-mail: marcone.oliveira@ufjf.edu.br
Um dos tipos possíveis de FE é a fase estacionária monolítica (FEM), a qual pode ser definida como um leito formado por uma única estrutura, porosa, contendo pequenos domínios e canais relativamente grandes, que proporcionam alta permeabilidade e eficiência nas separações, resultante da polimerização do monômero. ${ }^{1,10}$ A FEM pode ser produzida através do processo sol-gel in situ, o qual se baseia na inserção da solução sol no interior do tubo capilar, passando ao estado de gel através de foto ou termopolimerização. A formação homogênea da FEM concomitante à sua inserção no tubo é a etapa mais crítica no processo de produção e requer cuidados e equipamentos convenientes. ${ }^{11,12}$ A reprodução na distribuição e tamanho dos poros no leito polimérico pode ser baixa nos dois tipos de polimerizações, se o controle das variáveis, ou seja, luz ou calor na etapa de polimerização não for realizada de maneira eficiente. Normalmente se recorrem a sistemas específicos, produzidos, especialmente, para promover incidência de radiação luminosa ${ }^{13}$ ou fornecimento de calor, uma vez que câmaras foto emissoras ou estufas convencionais apresentam certa limitação quanto a eficiência. Nesse sentido, a etapa de funcionalização também demanda de aparatos específicos capazes de proporcionar a efetiva transformação química entre os reagentes e solventes para a produção da FEM funcionalizada.

Levando-se em consideração pesquisas associadas ao desenvolvimento de dispositivos que auxiliam o processo de preparação de FE, de maneira geral, o presente trabalho aborda a construção de um mini forno para a funcionalização da FEM por termoativação. $\mathrm{O}$ dispositivo permite o aquecimento in situ do tubo capilar contendo no interior os reagentes de funcionalização, proporcionando a modificação química da FEM. Acreditamos, ainda, que o trabalho apresenta relevante potencialidade para grupos interessados na preparação das FEM mediada pela polimerização iniciada termicamente.

\section{EXPERIMENTAL}

\section{Reagentes e solventes}

Os reagentes e solventes utilizados no preparo das FEM e da funcionalização foram os seguintes: metacriloxipropiltrimetoxisilano (MPTMS-98\%) - Acros Organics (New Jersey, EUA); 3- mercaptopropiltrimetóxisilano- (SMPTMS-98\%) - Acros Organics (New Jersey, EUA); óxido de bis(2,4,6-trimetilbenzoil)-fenilfosfino (Irgacure 819) - Ciba (São Paulo, Brasil); tolueno (alto grau de pureza- 99,5\%) - Beckman (Fullerton, EUA); metanol - Quimex 
(Tubarão, Brasil- 98,8\%); ácido clorídrico - Vetec (Rio de Janeiro, Brasil-37\%); Ácido tetracloro áurico pentahidratado - Acros Organics (New Jersey, EUA).

\section{Seringas e conectores}

Seringas Gastight de $25 \mu \mathrm{L}$ (1702 RNR) e $50 \mu \mathrm{L}$ (1705 TLL) foram obtidas da Hamilton Company, Reno, Estados Unidos. As seringas são acopladas aos capilares pela agulha, com o auxílio de conexões PEEK (polímero PEEK ${ }^{\mathrm{TM}}$, marca registrada de VictrexPlc.): uniões True-ZDV para micro luvas (P-720); adaptadores do tipo Luer-to-MicroTight ${ }^{\circledR}$ (P-662); luvas tubulares do tipo MicroTight ${ }^{\circledR}$ Green PEEK (F-185X); luvas tubulares tipo Micro Tight ${ }^{\circledR}$ Black PEEK (F-186X); conectores padrão para luvas tubulares do tipo MicroTight ${ }^{\circledR}(\mathrm{F}-125 \mathrm{x})$ e conectores padrão para tubos capilares de $360 \mu \mathrm{m}$ de diâmetro, MicroTight@Blue PEEK (F-124Sx).

\section{Capilares}

Capilares de sílica fundida revestidos externamente com fluoropolímero de Teflon ${ }^{\circledR}$ transparente no UV profundo, da série TSU, com dimensões de $100 \mu \mathrm{m}$ d.i. e $375 \mu \mathrm{m}$ d.e. foram importados da Polymicro Technologies (Phoenix, EUA).

\section{Síntese das nanopartículas de ouro}

A síntese das nano partículas de ouro (AuNps) foi baseada no método descrito por Turkevitch et al. ${ }^{13}$ no qual $2,5 \times 10^{-6}$ mol do ácido tetracloro-áurico-trihidratado $3 \cdot \mathrm{H}_{2} \mathrm{O} \mathrm{H}\left[\mathrm{AuCl}_{4}\right]$ foi submetido a dissolução em água deionizada em um balão volumétrico de $10 \mathrm{~mL}$. A solução de coloração amarela foi agitada e aquecida até a ebulição. Em seguida, adicionou-se $0,75 \mathrm{~mL}$ de citrato de sódio - $\mathrm{C}_{6} \mathrm{H}_{5} \mathrm{Na}_{3} \mathrm{O}_{7}$ $0,5 \% \mathrm{~m} / \mathrm{v}$, que agiu como estabilizante e redutor do ouro, promovendo a redução de $\mathrm{Au}^{+3}$ para $\mathrm{Au}^{0}$. Logo após o resfriamento e a mudança da coloração de amarelo para violeta, o que indica o fim da reação, foram adicionados $2,5 \mu \mathrm{L}$ do passivante de quitosana $0,001 \mathrm{~mol} \mathrm{~L}^{-1}$.

\section{Funcionalização das FEM}

A FEM foi preparada de acordo com trabalhos publicados por Vaz et al., ${ }^{14-16}$ através da foto polimerização. Nessa preparação é realizada a ativação dos grupos silanóis da parede de sílica do capilar com o uso de soluções contendo hidróxido de sódio $0,1 \mathrm{~mol} \mathrm{~L}^{-1}$ seguida por ácido clorídrico também $0,1 \mathrm{~mol} \mathrm{~L}^{-1}$, ambos por trinta minutos. Por fim é realizada a limpeza com metanol e a FEM é seca com passagens seguidas de ar, sendo todas as soluções percoladas com o auxílio do dispositivo manual a alta pressurização- DMAP. ${ }^{12}$

Depois do preenchimento do bulk do capilar com a FEM, ocorre a etapa de pré-funcionalização, baseada no trabalho de Fang-Gui et al., ${ }^{17}$ no qual o reagente sililante de pré-funcionalização 3-mercaptopropilsilano (MPTMS) é submetido a diluição em tolueno na proporção 1/9. Em seguida, o reagente é percolado por dentro da FEM a temperatura de $110{ }^{\circ} \mathrm{C}$, durante uma hora. Contudo, nessa etapa, segundo a referência seguida, não foi descrito como ocorreu o aquecimento no momento da reação entre o agente sililante e a FEM.

A pré-funcionalização serve para garantir a ancoragem das nanopartículas na FEM no momento da funcionalização, já que o agente de pré-funcionalização usado apresenta grupos tiois em sua estrutura que podem ligar-se eletrostaticamente e ou sob diferentes formas, com as AuNps. ${ }^{18} \mathrm{Na}$ etapa final ocorre a funcionalização, ou seja, a solução das AuNps é percolada por uma hora a temperatura ambiente pela FEM pré-funcionalizada. ${ }^{17}$

\section{RESULTADOS E DISCUSSÕES}

\section{Considerações sobre a montagem do mini forno}

O mini forno, conforme mostra o esquema da Figura 1, é constituído por dois tubos concêntricos, o mais externo (B) feito de tubo de PVC e o mais interno (C) de cobre. Entre os dois tubos inseriu-se lã de vidro (A) para reduzir a transferência de calor do tubo de cobre para o tubo de PVC. A excentricidade do tubo de cobre é ajustada pelos três parafusos centralizadores (I), localizados nas extremidades do tubo de PVC. O controle dessa excentricidade é importante, pois isso garante que a dissipação de calor seja uniforme ao longo da parte radial e da extensão do tubo. O tubo de cobre é coberto por uma camada de teflon (D). A função da camada de teflon é isolar elétrica e termicamente o tubo de cobre. Além de isolar eletricamente, o teflon também é capaz de suportar as altas temperaturas geradas pela passagem da corrente elétrica no fio de níquel-cromo. Sobre a camada de teflon é enrolada uma resistência elétrica de níquel-cromo(E), que tem a função de produzir calor para o sistema. A resistência é conectada a uma fonte de corrente elétrica. Dentro do tubo de cobre é inserido um capilar de sílica com diâmetro de $0,52 \mathrm{~mm}(\mathrm{~F})$. Para garantir que o capilar permaneça no centro do tubo de cobre foi utilizado discos de teflon (H) com o diâmetro igual ao diâmetro interno do tubo de cobre. A função desses discos, além de manter o capilar no centro, é evitar a transferência de calor por convecção na direção longitudinal do tubo, pois dessa forma é possível criar pequenas regiões com temperaturas quase estacionárias. Isto garante a uniformidade da temperatura ao longo do tubo. Dentro do microcapilar (G), ou seja, a coluna capilar de sílica fundida, são inseridos os reagentes para a termoativação ou termopolimerização. Assim, ao controlar a amperagem gerada pela fonte é possível obter a temperatura desejada, atingindo um regime estacionário da temperatura e ao mesmo tempo garantindo sua uniformidade ao longo de toda a extensão do tubo. O comprimento total do tubo de PVC é de $42 \mathrm{~cm}$ e o de cobre $40 \mathrm{~cm}$.

Na Figura 2 é apresentada a foto do sistema completo montado contendo a fonte de tensão, um dos medidores de temperatura e tubo PVC, apresentando internamente os dispositivos e externamente os fios e conectores. O controle da temperatura é registrado através de três sensores de temperatura. Um sensor de temperatura é um termômetro digital comercial posicionado na entrada do forno, veja Figura 2B. Esse termômetro fornece a temperatura entre o tubo de cobre e a camada de teflon. Os outros dois sensores são termoresistores inseridos entre o capilar de sílica e o tubo de cobre. Estes sensores de temperatura foram posicionados a uma distância de 3/10 e 7/10 com relação a entrada do mini forno. Estas distâncias foram selecionadas para coincidir exatamente entre dois gomos formados pelos discos de teflon. Na foto da Figura 3A é possível observar 5 discos de teflon. Quando esse sistema é inserido dentro do tubo de cobre resulta na formação de 4 gomos ou bolsas de ar. Um termoresistor ficou posicionado entre o primeiro e o segundo disco de teflon e o outro termoresistor entre terceiro e quarto disco. Os termoresistores são de tamanho reduzido $\left(1 \mathrm{~mm}^{2}\right.$ de área). Este tamanho permite manter todo o sistema fechado, evitando a perda de calor. A medida da temperatura por esses dois termoresistores permitem analisar a estabilidade da transferência de calor no primeiro e terceiro gomo. Cada termoresistor faz parte de um divisor de tensão e a medida dessa tensão permite determinar a temperatura. Vale ressaltar que os discos de teflon além de manter o tubo capilar no centro também servem para reduzir efeitos de transferência de calor por convecção.

As fotos apresentadas na Figura 3 mostram alguns detalhes das partes do mini forno. A foto da Figura 3A apresenta detalhes da parte mais interna com o capilar de sílica conectado aos discos de teflon (tubo mais estreito à esquerda na foto), que são posteriormente introduzidos 
dentro do tubo de cobre (tubo à direita na foto). O tubo de cobre é revestido por uma camada de teflon com a função de isolar eletricamente a resistência de níquel-cromo. Pela foto da Figura 3B é possível observar a estrutura interna e o ajuste para centralizar o tubo de cobre.

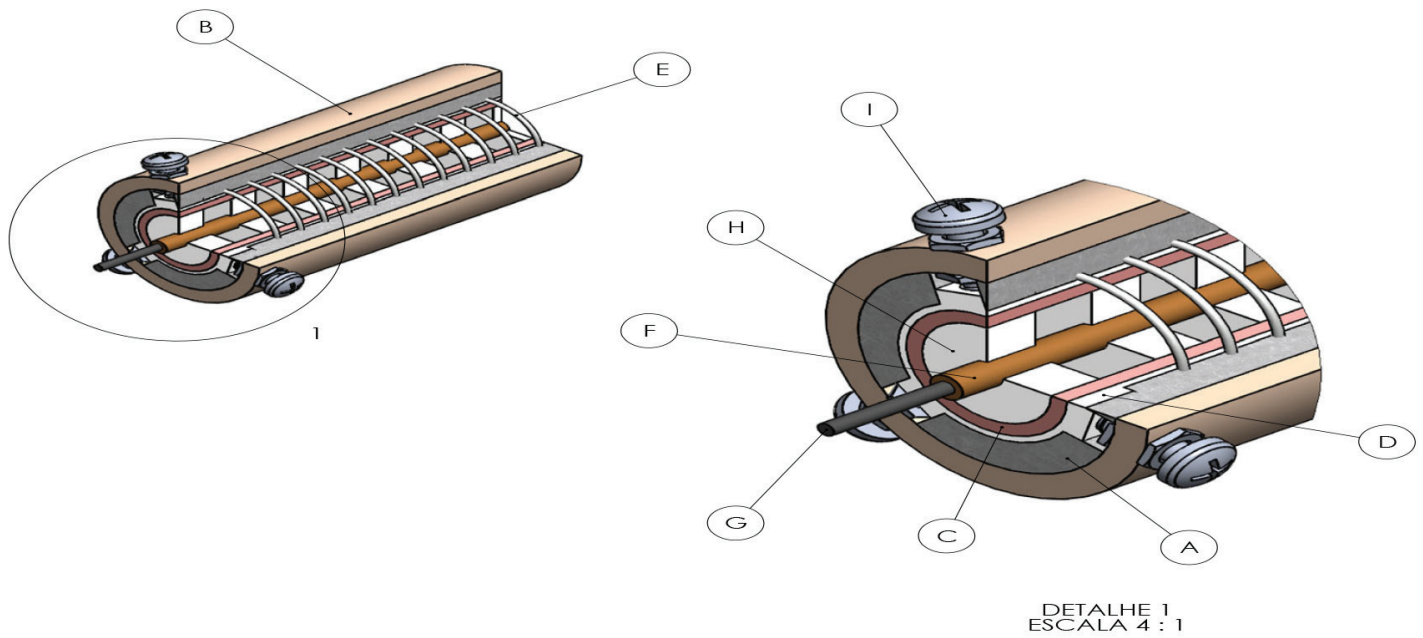

Figura 1. O desenho mostra a estrutura interna geral do mini forno. A figura da direita é uma ampliação da região 1 da esquerda. As principais partes são: $A$ - Lã de vidro, $B$ - tubo de PVC, $C$-Tubo de cobre, $D$ - Teflon, $E$ - fio níquel-cromo, $F$ - capilar de sílica, $G$ - micro capilar, $H$-Disco de Teflon, $I$ - parafuso centralizador

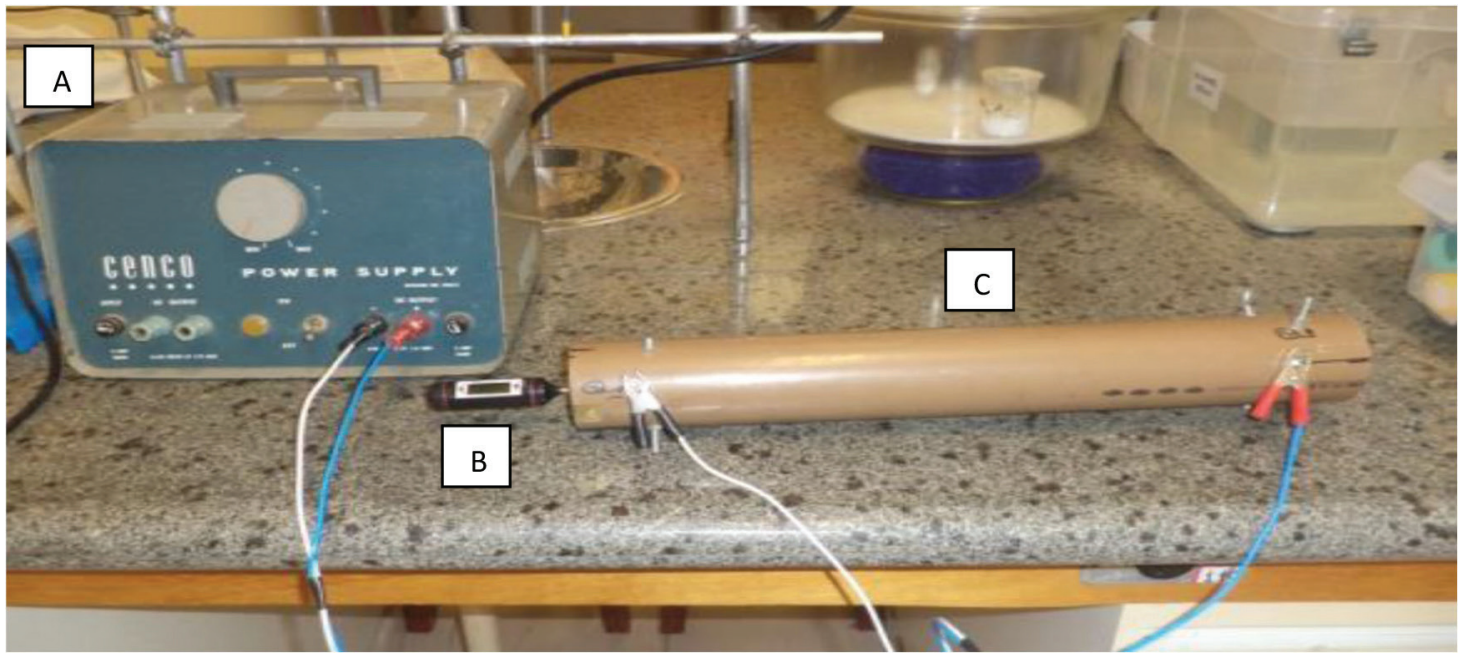

Figura 2. Foto do mini forno completo com fonte de corrente conectada (A), termômetro digital (B) e tubo PVC (C).As extremidades do tubo estão fechadas para evitar transferência de calor
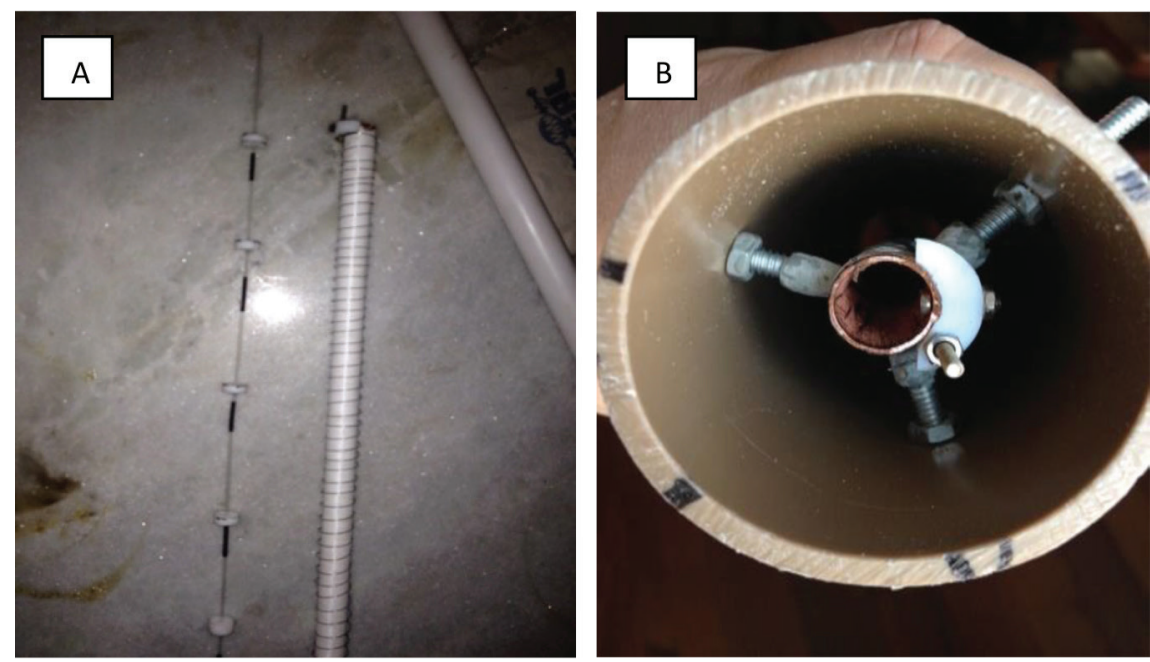

Figura 3. Fotos de algumas partes do mini forno: (A) capilar de sílica conectado aos discos de teflon (tubo mais à esquerda na foto), que são introduzidos dentro do tubo de cobre (tubo à direita na foto) e que ainda é revestido por uma camada de teflon. (B) estrutura interna e o ajuste para centralizar o tubo de cobre 


\section{Testes de monitoramento da temperatura do mini forno}

Na Figura 4 está apresentado o gráfico obtido com os dados experimentais da medida da temperatura em dois pontos diferentes ao longo do tubo do mini forno. Os dois sensores ficaram posicionados no centro do tubo, ou seja, próximo ao capilar a uma distância de 3/10 e 7/10 da entrada do tubo. As distancias 3/10 e 7/10 correspondem ao primeiro e terceiro gomos. Também foi fixado um termômetro em uma das extremidades do tubo, vide Figura 2B. Através do controle da corrente elétrica que atravessa o fio de níquel-cromo (Figura $3 \mathrm{~A}$ ) é possível também controlar a temperatura do mini forno. O sistema permite variar a temperatura na faixa de 30 a $180^{\circ} \mathrm{C}$. No experimento realizado a temperatura selecionada foi de $130{ }^{\circ} \mathrm{C}$. No gráfico da Figura 4 está o registro da temperatura em função do tempo de dos dois sensores localizados a 3 e 7 décimos do comprimento do tubo. Pode-se observar que a estabilização da temperatura foi alcançada a partir de 6 minutos, para uma variação da temperatura registrada de $130 \pm 1,0{ }^{\circ} \mathrm{C}$. Estas medidas corroboram com o fato de que o mini forno possibilita a termopolimerização da fase estacionária, posto que a distribuição da temperatura é uniforme ao longo do tubo após 6 minutos.

\section{Aplicação do mini forno para a funcionalização térmica de FEM}

Em função das necessidades inerentes a funcionalização da FEM ancorada na superfície interna da coluna capilar, emergiu a necessidade de se construir um equipamento específico para o aquecimento, no momento da passagem do reagente sililante, o qual uma vez aderido, os sitios ativos são utilizados para ligar as AuNps à FEM. Nesse sentido, foi desenvolvido um aparato de aquecimento que garanta uniformidade do aquecimento em regime estacionário. No caso em questão, as AuNps ficaram aderidas aos grupos tiois do reagente sililante contido no monolito, o que pode ser evidenciado pela mudança da coloração da FEM que passa de incolor ao violeta no término do processo. A visibilidade da coloração violeta aumenta de acordo com a maior concentração das nanopartículas ancoradas à FEM. Para alcançar a funcionalização, o capilar contendo a FEM foi inserido no interior do mini forno aquecido. Em seguida o reagente sililante foi introduzido, através do DAP, no interior da coluna capilar contendo a FEM, permanecendo em contato pelo período de 1 hora sob temperatura de $100{ }^{\circ} \mathrm{C}$. Na Figura 5 é possível observar, após a limpeza da FEM no equipamento de EC, o resultado do processo de funcionalização através de um microscópio de bancada com baixa resolução. As AuNps no interior do capilar superior (A), a qual

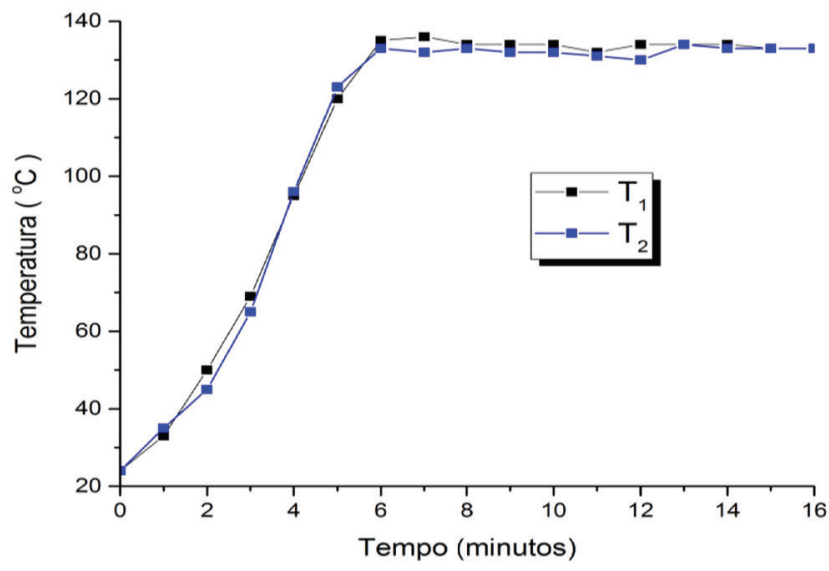

Figura 4. Gráfico de temperatura versus tempo, obtidos pelos dois sensores (T1 e T2). O comprimento total do tubo é de $40 \mathrm{~cm}$ e os sensores foram posicionados em 12 e $28 \mathrm{~cm}$

apresenta aspecto longitudinal homogêneo, estruturas bem visíveis e coloração violeta nítida em comparação com o capilar inferior (B) na ausência de funcionalização.

A comprovação formal da funcionalização da FEM foi mediada pela análise elementar de CHNS, a qual resultou como resposta a adesão do enxofre de $1,94 \%$. É relevante destacar que as medidas de CHNS foram realizadas a partir de FEM com massas iguais, tendo como parâmetro um testemunho em branco, no qual a FEM foi analisada na ausência de funcionalização, ou seja, sem a passagem do reagente sililante. Constatou-se, através da análise elementar a presença de apenas $0,12 \%$ de enxofre na sua estrutura.

É importante ressaltar que exaustivas tentativas de termo funcionalização da FEM através do aquecimento mediado por estufas não proporcionaram resultados eficazes e eficientes. Na Figura 6 é possível observar a comparação entre FEM funcionalizadas sem o uso do mini forno (A) e outra FEM preparada com o uso do equipamento de aquecimento desenvolvido (B). É importante destacar que a imagem da Figura 6 (B), foi obtida após corrida cromatográfica no equipamento de EC.

Na Figura 7, está apresentado a imagem, ainda com o microscópio de bancada com baixa resolução, de corte transversal de duas FEM para uma mais favorável visualização da funcionalização com AuNps realizada com o uso do mini forno (A) em comparação com uma FEM isenta de nanopartículas (B).

Na Figura 8, é apresentado a imagem do capilar funcionalizado obtida por Microscópio Eletrônico de Varredura (MEV), acoplada

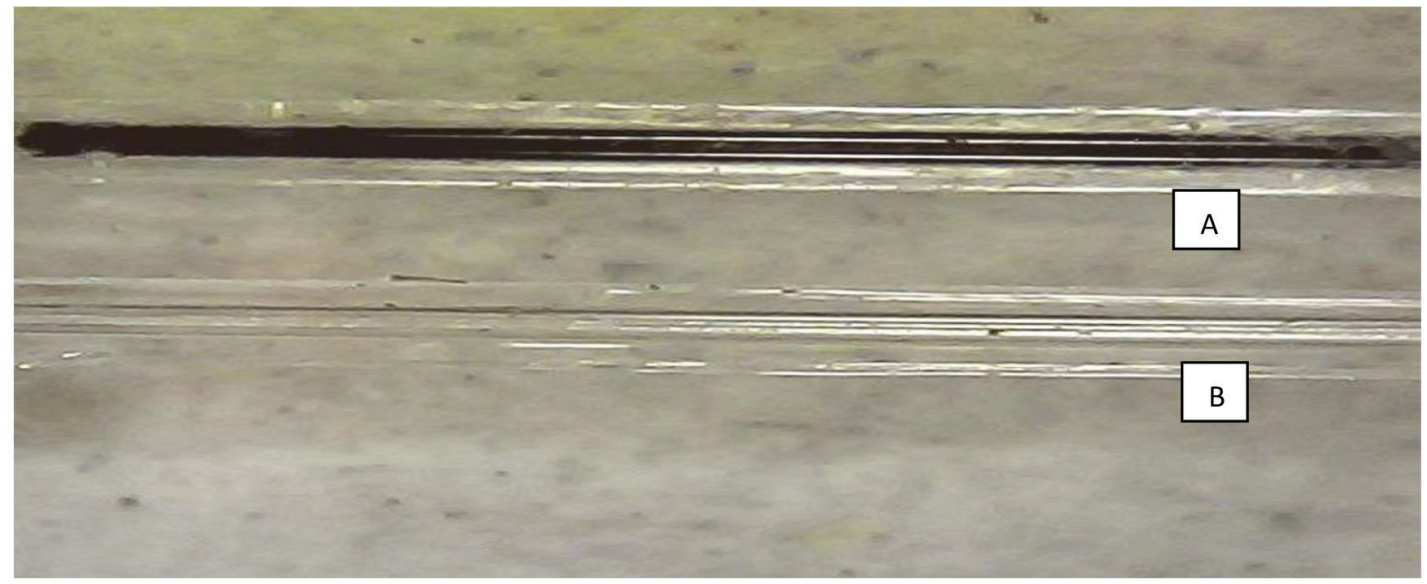

Figura 5. FEM com alta adesão de AuNps devido ao uso do mini forno (A) e FEM sem o reagente sililante e consequentemente sem a funcionalização com AuNps (B) 

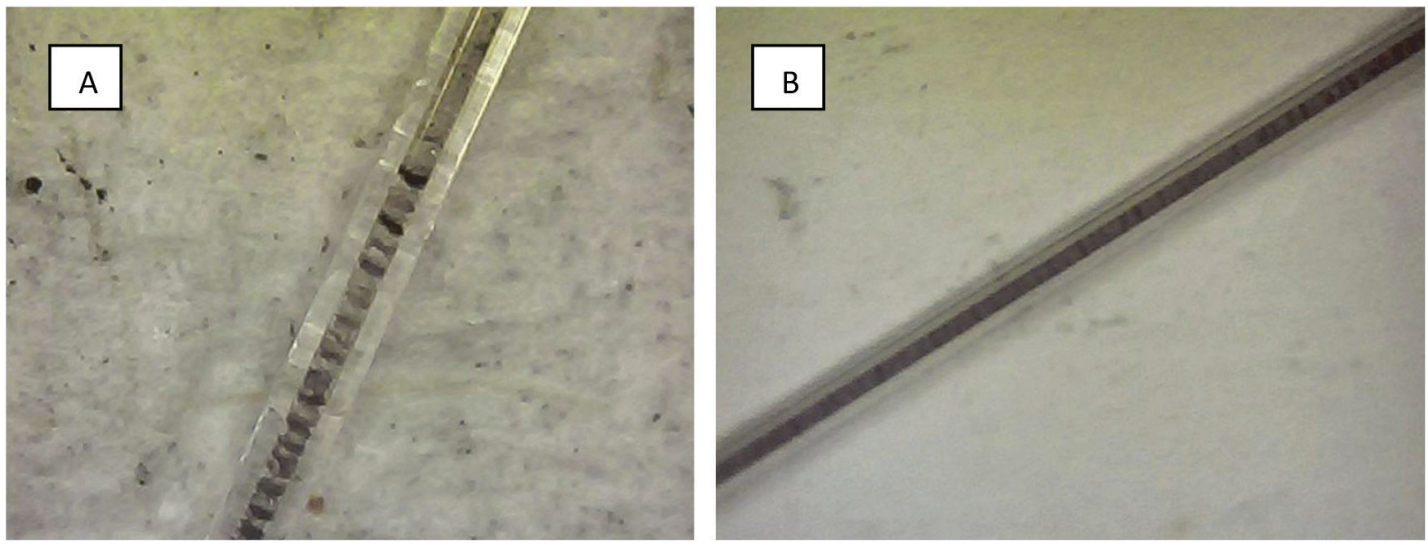

Figura 6. Capilar mediado por estufa convencional (A), falhas na funcionalização, adesão das AuNps em apenas alguns pontos, conforme análise da coloração Legenda (B) FEM produzida pelo mini forno, aspecto homogêneo de coloração violeta em toda a extensão
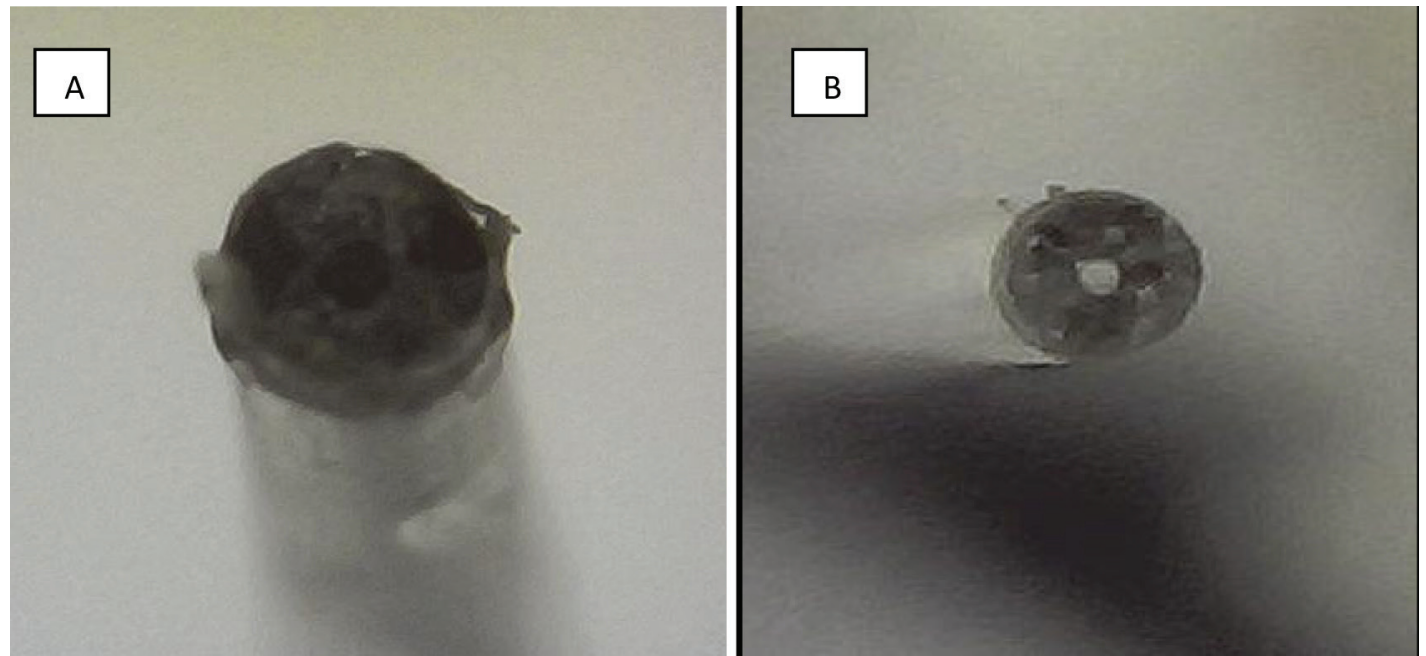

Figura 7. Corte radial das FEM com funcionalização após uso do mini forno (A) e sem funcionalização, apenas com o polimero na parte central do capilar (B)

com Espectroscopia por Dispersão de Energia de Raios X (EDS). A partir da análise mediada por MEV e EDS pôde- se concluir que a funcionalização da FEM foi bem sucedida com o uso do mini forno. Nessa técnica de microanálise qualitativa e semi quantitativa, além da investigação morfológica da superfície, é fornecida a composição dos elementos químicos distribuídos no material, ou seja, os materiais eletroativos presentes no capilar com a FEM funcionalizada. ${ }^{19} \mathrm{Nesse}$ sentido, na Figura (A) a coloração violeta referente as AuNps aderidas ao monolito é constatada na parte central do interior do capilar, assim como no espectro da Figura 8 (B), através do EDS, confirma-se a presença do teor de ouro, além de fluor, alumínio, carbono e oxigênio.

\section{Aplicação das FEM em ECC}

Os eletrocromatogramas apresentados abaixo na Figura 9, para a separação de HPAs, representam FEM antes da funcionalização.
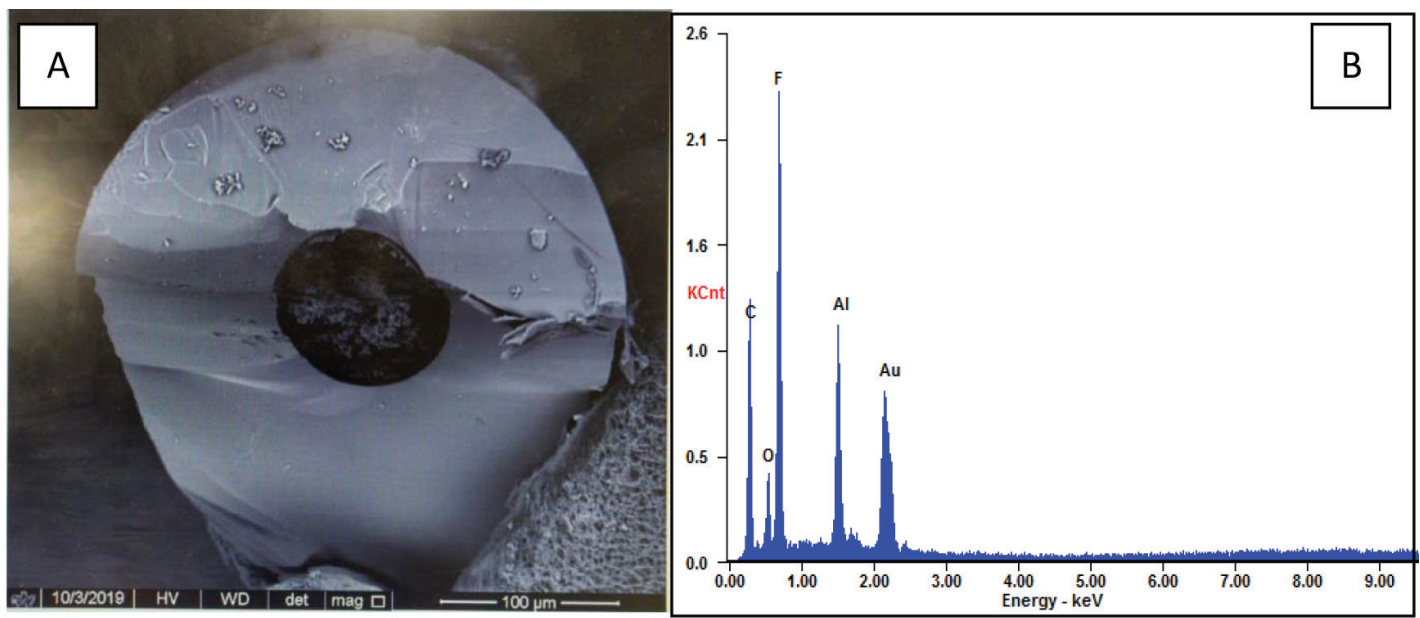

Figura 8. MEV da FEM funcionalizada no interior do capilar de sílica (A) e espectro de EDS para a área analisada do capilar de TSU com FEM funcionalizada com AuNps 

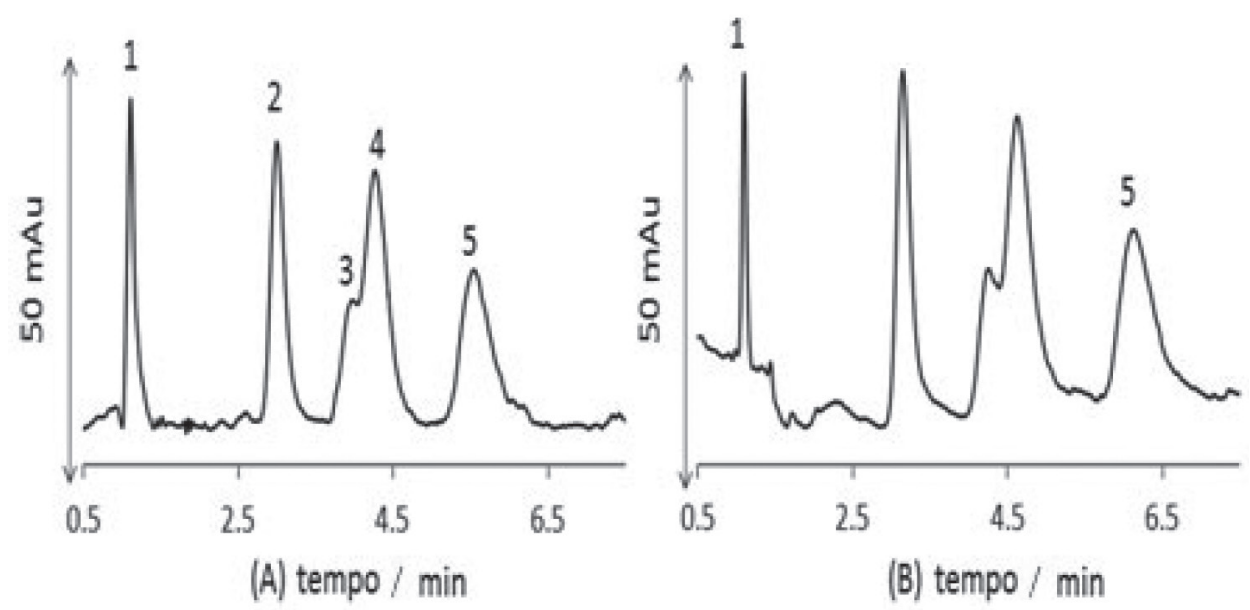

Figura 9. Eletrocromatogramas obtidos em colunas produzidas A, B, C e D em capilar TSU Ordem de eluição: (1) tioureia, (2) naftaleno, (3) acenafteno, (4) fluoreno e (5) fenantreno, diluídos em metanol a 1,0 mmol/L. FM: $\mathrm{NH}_{4} \mathrm{Ac}$ 16,7 mmol/L (60\%) e acetonitrila (40\%); voltagem: $-20 \mathrm{kV}$; temperatura: $20{ }^{\circ} \mathrm{C}$; injeção: -25 mbar por $5 \mathrm{~s}$; detecção: $220 \mathrm{~nm}$
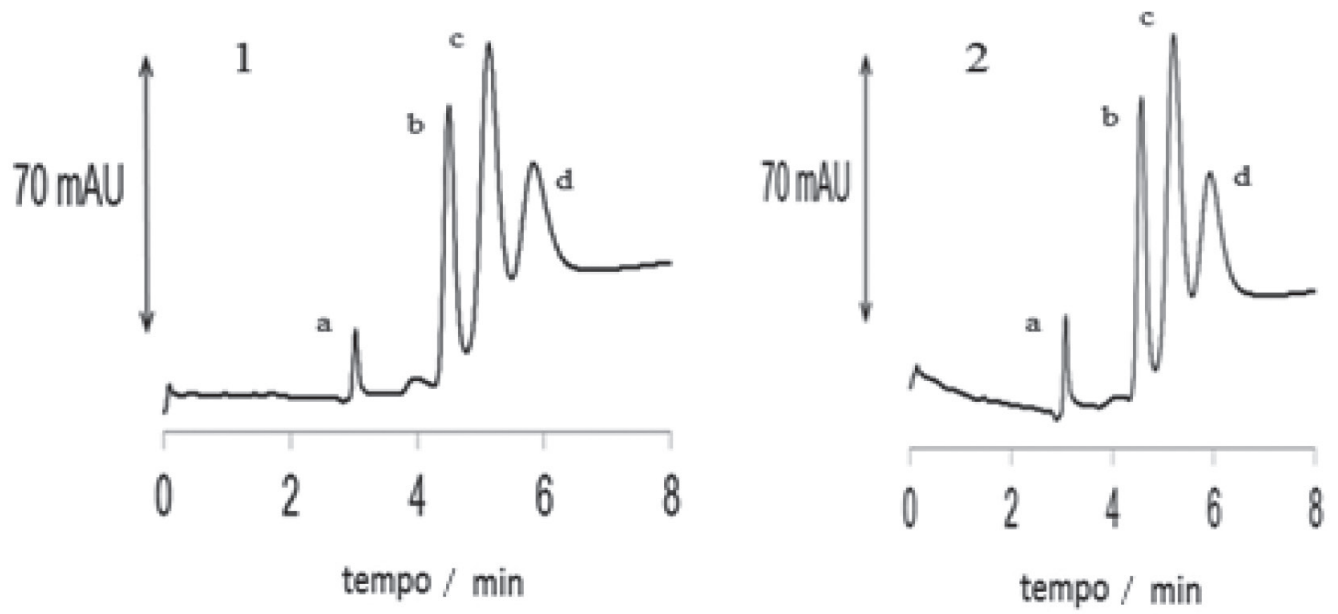

Figura 10. FEM- (1 e 2) funcionalizada no mini forno com AuNps. Eletrocromatogramas obtidos em uma coluna produzida em capilar TSU em análise com: (a) tioureia (0,1 mmol/Lem metanol), (b) naftaleno, (c) acenafteno e (d) fluoreno, diluídos em metanol a 1,0 mmol/L. FM: $\mathrm{NH}_{4} \mathrm{Ac}$ 16,7 mmol/L (60\%) e acetonitrila (40\%); voltagem: $-20 \mathrm{kV}$; temperatura: $20^{\circ} \mathrm{C}$; injeção: -25 mbar por $5 \mathrm{~s}$; detecção: $220 \mathrm{~nm}$

Pode-se observar pronunciada coeluição entre o acenafteno (3) e o fluoreno (4).

A seguir são mostrados eletrocromatogramas com HPAs em duas FEM funcionalizadas com as AuNPs, Os eletrocromatogramas apresentados na Figura 10 foram obtidos a partir de colunas preparadas, independentemente, com o mesmo método, porém com o uso do mini forno no auxílio da funcionalização. Através da ilustração pode-se observar o maior tempo de retenção dos HPAs, o que sinaliza positivamente para a ocorrência na mudança da superfície dos poros por onde percola os analitos. Com isso, houve separação de maneira mais eficiente dos picos do acenafteno (c) e fluoreno (d) o que pode contribuir com estudos futuros sobre a arte da produção e aplicação das FEM funcionalizadas com o benefício do mini forno.

\section{CONCLUSÃO}

O estudo realizado apresenta a construção de um aparato de aquecimento, capaz de auxiliar de forma eficiente o aquecimento na pré-funcionalização das FEM em colunas capilares com a AuNps, o que pôde ser evidenciada pela mudança de coloração, do branco para o violeta, característica de adesão das nanopartículas à FEM. É importante ressaltar que o dispositivo construído apresenta grande potencial para aplicação na termopolimerização de fases estacionárias monolíticas em colunas capilares de sílica fundida.

\section{REFERÊNCIAS}

1. Faria, A.M.; Bottoli, C. G. B.; Jardim, I. C. S. F.; Collins, C. H.; Quim. Nova 2006, 29, 300.

2. Svec, F.; Huber, C. G.; Anal. Chem. 2006, 78, 2101.

3. Zou, H.; Huang, X.; Ye, M.; Luo, Q.; J. Chromatogr., A 2002, 954, 5.

4. Tavares. M. F.; Quim. Nova 1996, 19, 173

5. Shediac, R.; Ngola, S. M.; Throckmorton, D. J.; Anex, D. S.; Shepodd, T. J.; Singh, A. K.; J. Chromatogr. A 2001, 925, 251

6. Bandilla, D.; Skinner, D. C.; J. Chromatogr. A 2003, 1004, 167.

7. Haixia, L.; Wang, J.; Wang, X.; Lin, X.; Wu, X.; Xie, Z.; J. Pharm. Biomed. Anal. 2007, 43, 352.

8. Kato, M.; Sakai, K.; Toyo'oka, T.; Dulay, M. T.; J. Chromatogr. A 2002, $961,45$.

9. Lammerhofer, M.; Svec, F.; Frechet, J. M. J.; Lindner, W.; Trends Anal. Chem. 2000, 19, 676; Lammerhofer, M.; Svec, F.; Frechet, J. M. J.; Lindner, W.; J. Microcolumn Separations 2000, 12, 597.

10. Svec, F.; Peters, E. C.; Sykora, D.; Frechet, J. M. J.; J. Chromatogr. A 2000, 887,3 .

11. Ponce, M. J. S. G.; Tese de Doutorado, Universidade Estadual de Campinas, Brasil, 2011. 
12. Ribeiro, S. P.; Vaz, F. S. A.; Oliveira. M. A. L.; Quim. Nova 2015, 38, 132.

13. Turkevich, J.; Stevenson, P. C.; Hillier, J.; Disc. Faraday Soc. 1951, 11, 55.

14. Vaz, F. A. S.; de Queiroz, M. P. G.; Ribeiro, S. J. L.; Quim. Nova 2008, 31, 2156.

15. Vaz, F. A. S.; Castro, P.; Molina, C.; Oliveira, M. A. L.; Talanta 2008, $76,226$.
16. Vaz, F. A. S.; Moutinho, A.; Mendonça, J.; Oliveira, M. A. L.; Microchem. J. 2012, 100, 21

17. Fang-gui, Y.; Jun-Yu, L.; Yuan, W., Ai-Zhu, Z.; Jian-Niao, T.; Shu-Lin, Z.; Chin. J. Anal. Chem. 2011, 3, 39.

18. Subramaniam, V.; Griffitha, L.; Haes, A. J.; Analyst 2011, 136, 3469.

19. Martins, P. R.; Rocha, M. A.; Angnes, L.; Toma, H. E.; Araki, K.; Electroanalysis 2011, 23, 2541. 